\title{
COMMUNICATIONS
}

\section{ETUDE DE TRANSFERT DE POLLUTION PAR TEMPS DE PLUIE SUR LE RESEAU D'ASSAINISSEMENT DU DISTRICT URBAIN D'ARRAS}

\author{
L. HERREMANS *, C. ZOBRIST *, M. CHAUMON- \\ TET * \\ *Anjou-Recherche \\ ** Compagnie Générale des Eaux
}

\section{RESUME}

Dans le cadre des études menées par le District Urbain d'Arras en collaboration avec l'Agence de l'Eau Artois-Picardie sur le fonctionnement des réseaux d'assainissement districaux, une étude de transfert de pollution par temps de pluie a été réalisée à l'aide du logiciel FLUPOL [1], [2]. 
Après avoir réalisé la validation du modèle, des simulations effectuées à partir de pluies de projet ont permis d'évaluer les flux de pollution déversés dans le milieu récepteur.

Ainsi, lors d'épisodes pluvieux habituels (période de retour trimestrielle), les déversements dans la Scarpe représentent de l'ordre de $80 \%$ de la pollution globale (eaux usées et eaux pluviales) produite par l'agglomération.

\section{OBJECTIFS DE L'ETUDE}

Les objectifs de l'étude sont:

- de quantifier l'importance en volumes et en charges de pollution des différents rejets (déversoirs d'orage, rejets directs) ;

- d'analyser, sur la base des pluies de projet, l'intérêt de la mise en place de bassins de stockage afin de limiter la pollution rejetée :

- de définir les aménagements prioritaires à réaliser sur le réseau et à la station d'épuration.

Afin de mener à bien cette étude, il est apparu nécessaire de passer par la modélisation mathématique en utilisant le modèle FLUPOL, mis au point conjointement par la Compagnie Générale des Eaux, l'Agence de l'Eau Seine-Normandie et le Syndicat des Eaux d'lle-de-France. te :

Dans ce cadre, la méthodologie adoptée a alors été la suivan-

- réalisation de campagnes de mesures quantitatives et qualitatives permettant de traduire, en chiffres, la réalité du terrain en certains points clés du réseau, et pour quelques événements pluvieux :

- utilisation du logiciel de modélisation, validé à partir des événements mesurés, par les pluies-types représentant les phé. nomènes contre lesquels on souhaite se protéger, afin d'évaluer les quantités de pollution résultant de ces événements et de définir les aménagements prioritaires à réaliser.

\section{LE MODELE FLUPOL}

Le modèle FLUPOL permet de simuler, en tout point d'un réseau d'assainissement desservant un bassin versant urbain en un système unitaire ou séparatif, les hydrogrammes et les flux de pollution générés par un événement pluvieux quelconque et caractérisés par un certain nombre de variables d'état. II tient compte d'une succession de phénomènes allant de l'accumulation des dépôts sur le sol à la remise en suspension éventuelle des sédiments dans les conduites.

Par ailleurs, il convient de souligner que le modèle utilisé sur le réseau districal n'a pas été calé de manière spécifique sur le site mais a été simplement validé.

\section{LE SITE D'ETUDE}

La superficie totale prise en compte dans cette étude est de 3.320 hectares et le linéaire des réseaux d'assainissement correspondant de 375 kilomètres.

L'ensemble des réseaux d'assainissement de type unitaire ou " eaux usées " aboutit à la station du District Urbain d'Arras, située sur la rive droite de la Scarpe à St-Laurent-Blangy.

Le découpage de ce territoire d'étude a permis de déterminer huit grands bassins-versants que nous dénommerons par la suite " secteurs". Par ailleurs, le réseau modélisé a été bien évidemment simplifié par rapport au réseau complet et seuls les axes principaux ont été pris en compte.

Les caractéristiques principales des huit secteurs sont regroupées dans le tableau 1.

\section{LA VALIDATION DU MODELE}

La validation du modèle a été réalisée par comparaison avec des mesures de débit et de pollution faites sur les deux principaux collecteurs du District (les collecteurs Boulevard et Crinchon).

Cette validation a été réalisée sur une pluie mesurée le 28.10.89 qui a représenté une hauteur précipitée de $4,4 \mathrm{~mm}$. Les principaux résultats de cette validation sont les suivants et sont récapitulés dans le schéma ci-après.

\subsection{LES MATIERES EN SUSPENSION}

Les charges polluantes et simulées dont apparaître des variations de :

- $16 \%$ pour le collecteur du Crinchon,

- $5 \%$ pour le collecteur des Boulevards.

Tableau 1: Caractéristiques principales des huit secteurs.

\begin{tabular}{|c|c|c|c|c|c|c|c|c|}
\cline { 2 - 9 } \multicolumn{1}{c|}{} & $\begin{array}{c}\text { O-N.O } \\
\text { Scarpe }\end{array}$ & $\begin{array}{c}\text { N.O } \\
\text { Scarpe }\end{array}$ & $\begin{array}{c}\text { N.Centre } \\
\text { Scarpe }\end{array}$ & $\begin{array}{c}\text { N.E } \\
\text { Scarpe }\end{array}$ & Tilloy.P & $\begin{array}{c}\text { S.O } \\
\text { Scarpe }\end{array}$ & Burien & Arras \\
\hline Superficie & 74 & 393 & 93 & 390 & 175 & 214 & 495 & 1.486 \\
\hline $\begin{array}{c}\text { Imp } \\
\text { Moyen }\end{array}$ & 0,2 & 0,21 & 0,48 & 0,2 & 0,22 & 0,24 & 0,31 & 0,31 \\
\hline $\begin{array}{c}\text { Densité } \\
\text { Ha }\end{array}$ & 7 & 17 & 80 & 10 & 4 & 15 & 29 & 29 \\
\hline $\begin{array}{c}\text { Nature du } \\
\text { réseau }\end{array}$ & Pluvial & Unitaire & Pluvial & Unitaire & Pluvial & Pluvial & Pluvial & $\begin{array}{c}\text { Unitaire } \\
\text { Séparatif }\end{array}$ \\
\hline $\begin{array}{c}\text { Linéaire } \\
\mathrm{m}\end{array}$ & 450 & 4.700 & 370 & 4.800 & 2.700 & 1.600 & 5.130 & 17.750 \\
\hline $\begin{array}{c}\text { Diamètre } \\
\text { canalisa- } \\
\text { tion }\end{array}$ & 800 à & 200 à & 1.000 & 1.500 & 200 à & 800 à & 1.000 à & 800 à \\
1.500 & $\begin{array}{c}500 \text { à } \\
\text { ouvrages } \\
\text { visitables }\end{array}$ \\
\hline
\end{tabular}




\subsection{LA DEMANDE CHIMIQUE EN OXYGENE (DCO)}

La comparaison des flux totaux mesurés et simulés donne les résultats suivants :

- $12 \%$ pour le collecteur du Crinchon,

$-20 \%$ pour le collecteur des Boulevards.

\subsection{L'AZOTE KJELDHAL}

Les écarts, concernant ce paramètre entre les charges polluantes mesurées et simulées, sont de:
$-18 \%$ pour le collecteur du Crinchon,

$-20 \%$ pour le collecteur des Boulevards.

Au vu de ces résultats, il a été considéré que le modèle était applicable au cas d'Arras. II était alors possible de passer à la phase prospective (figure 1).

\section{LA PHASE DE PROJET}

En accord avec l'Agence de l'Eau Artois-Picardie et le District d'Arras, il a été décidé de choisir une pluie de projet de caractéristiques suivantes: pluie uniforme de $10 \mathrm{~mm}$ de hauteur totale précipitée sur deux heures.

\section{PLUTE REELLE DU 28 OCTOBRE 19.89}

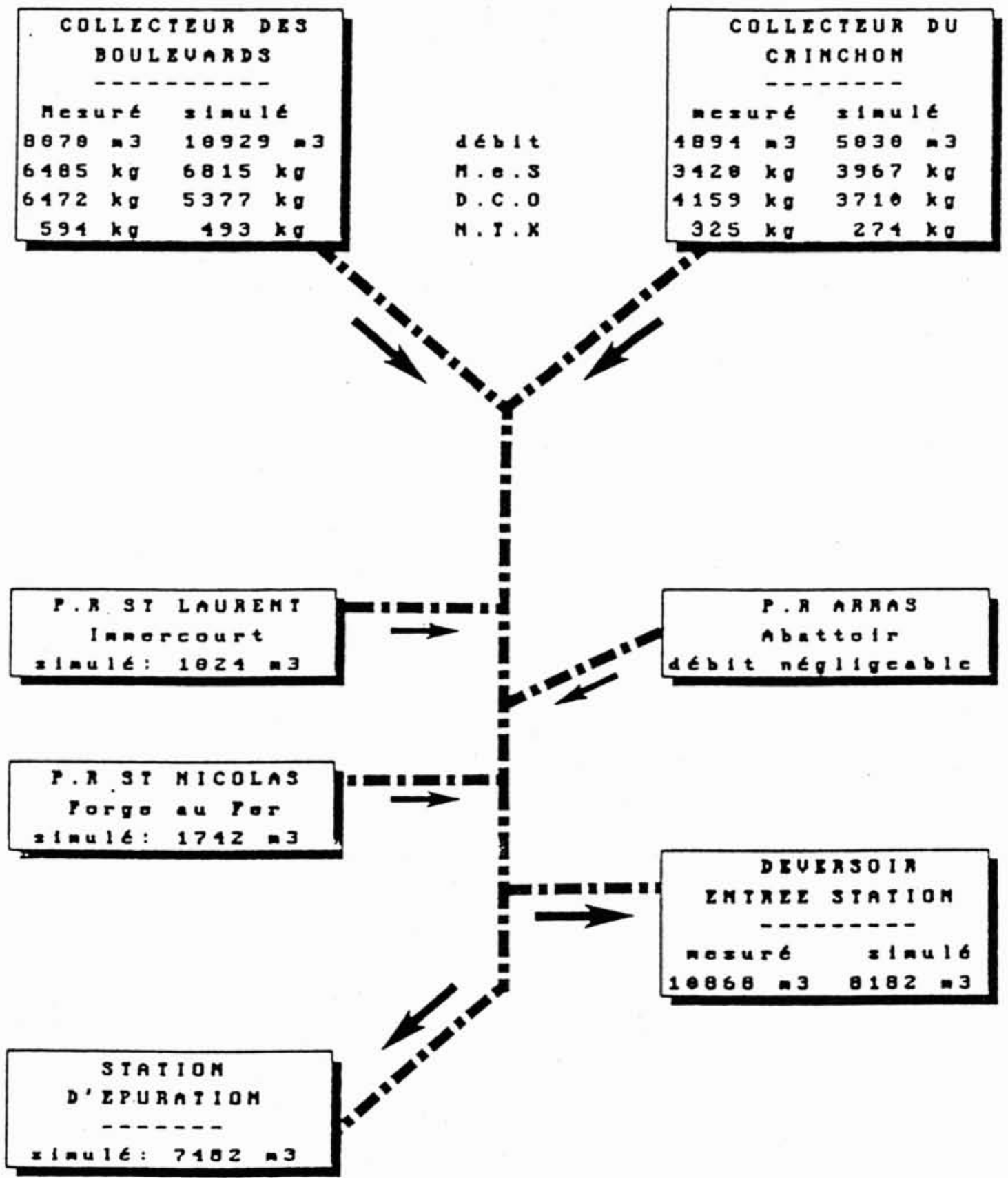

Figure 1: District urbain d'Arras. 
Cette pluie, d'occurence trimestrielle, peut être considérée comme non-exceptionnelle au sens de la directive C.E.E. du 21 mai 1991. Les résultats des simulations réalisées sur la base de cette pluie font apparaître que, globalement, les charges déversées sont :

- pour la DBO5 : de l'ordre de 6 tonnes,

- pour la DCO : de l'ordre de 30 à 40 tonnes,

- pour le NTK: de l'ordre de 1,5 à 2 tonnes.

Elles représentent, quelque soit le paramètre (DBO5, DCO ou NTK), de l'ordre de 80 à $85 \%$ de la pollution globale émise par le District (eaux usées + eaux de temps de pluie). On voit donc, que la pollution des rejets de temps de pluie, pour des pluies de fréquence non rare, est considérable et par ailleurs, est nettement supérieure à la pollution traitée en station d'épuration.

On note, par ailleurs, que le déversoir d'orage situé en tête de la station d'épuration représente, à lui seul, de l'ordre de $40 \%$ de la pollution totale déversée.

\section{CONCLUSION}

Les graphiques font apparaître qu'actuellement, pour la pluie de référence choisie et pendant la durée de l'écoulement:

- $92 \%$ de la charge totale en MES sont rejetés en Scarpe (soit près de 47 tonnes),

- $85 \%$ de la charge totale en DBO5 sont rejetés en Scarpe (soit près de 6 tonnes)

Tableau 2: Charges en MES (a), charges en DBO (b), charges en DCO (c) et charges en azote Kjeldahl (d).

Charges en M.E.S. en kg

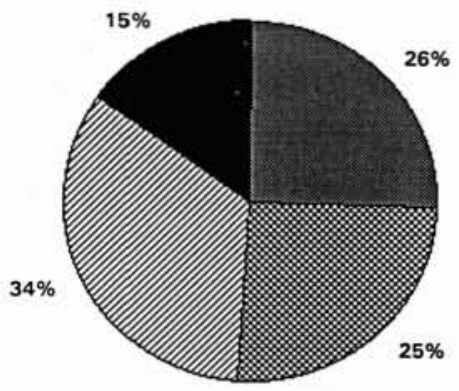

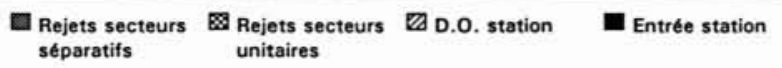

Charges en D.B.O. en kg

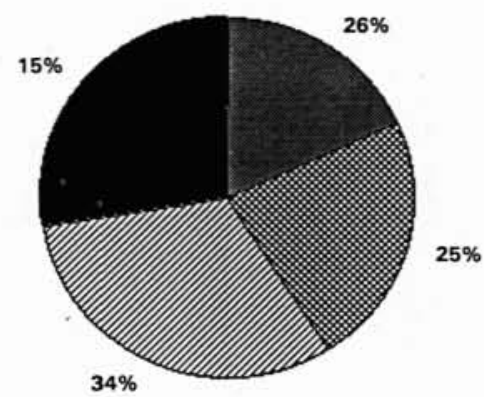
Rejets secteurs $\begin{aligned} & \text { Rejets secteurs } \\ & \text { séparatifs } \\ & \text { unitaires }\end{aligned}$
D.O. station

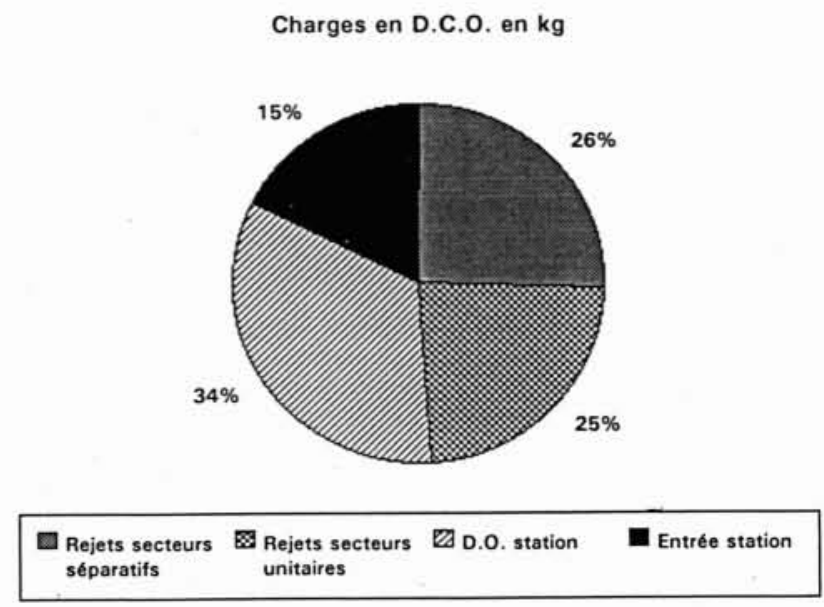

Charges en Azote Kjeldahl en kg

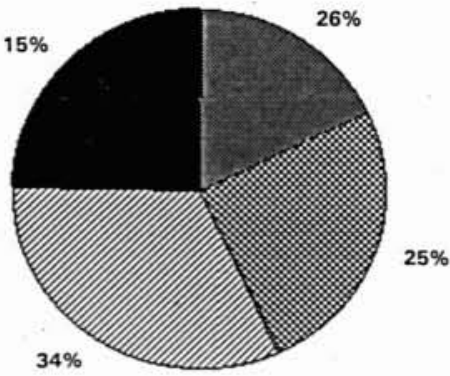

Rejets secteurs $\mathbb{Q}$ Rejets secteurs D.O. station séparatifs unitaires

- $90 \%$ de la charge totale en DCO sont rejetés en Scarpe (soit près de 33 tonnes),

$-86 \%$ de la charge en NTK sont rejetés en Scarpe (soit près de 2 tonnes)

Ces quelques chiffres montrent toute l'importance de la pollution rejetée par temps de pluie et la nécessité de prévoir les investissements permettant d'en diminuer les conséquences, afin que les travaux qui seront réalisés pour résorber les rejets de temps sec remplissent pleinement leur fonction.

Ces investissements doivent porter sur :

- réalisation de bassins de stockage temporaire avec envoi ultérieur vers la station d'épuration :

- réalisation de bassins de décantation et envoi des eaux décantées dans le milieu récepteur ;

- extension de la station d'épuration pour traiter les eaux de temps de pluie ;

- aménagement de certains déversoirs d'orage en vue de limiter les déversements (automatisation des seuils déversants).

\section{BIBLIOGRAPHIE}

[1] BUJON G. - Prévision des débits et des flux polluants transités par les réseaux d'égouts par temps de pluie : le modèle FLUPOL. La Houille Blanche, 1988, $n^{\circ}$ 1, pp. 11-23.

[2] BUJON G., HERREMANS L. - FLUPOL : modèle de prévision des débits et des flux polluants en réseau d'assainissement par temps de pluie. Calage et validation. La Houille Blanche, $1990, n^{\circ} 2$, pp. 123-139. 